\title{
Characterization of Human Cytosolic Thioredoxin Reductase by Site-specific Mutagenesis
}

\author{
Lin Yuan and Hakjung Kim* \\ Department of Chemistry, College of Natural Science, Daegu University, Kyoungsan 712-714, Korea \\ *E-mail: hjkim@daegu.ac.kr \\ Received September 6, 2010, Accepted October 4, 2010
}

Key Words: Thioredoxin reductase, Pyridine nucleotide-disulfide oxidoreductase, Selenoprotein, Dihydrolipoamide dehydrogenase, Site-directed mutagenesis

Thioredoxin reductase (EC 1.8.1.9) (TR) physiologically catalyzes the reduction of oxidized thioredoxin (Trx) at the expense of NADPH. The reduced Trx then can become an electron donor to diverse proteins such as ribonucleotide reductase, transcription factors, and Trx-dependent peroxidases, called peroxiredoxins. ${ }^{1}$ Trx is also important to various biological functions including immunomodulation, photosynthesis, apoptosis, and pregnancy in diverse organisms. TR belongs to the pyridine nucleotide-disulfide oxidoreductase family which includes dihydrolipoamide dehydrogenase (E3), glutathione reductase, mercuric reductase, and trypanothione reductase. ${ }^{2}$ Most of these enzymes, except E3, catalyze the transfer of electrons from NADPH to their specific substrates through FAD. Mammalian TRs are very different from those of bacterial origin, both in their structures and catalytic mechanisms. ${ }^{3}$ They are homodimeric proteins with subunit sizes of about $55 \mathrm{kDa}$ as shown in Figure 1, while those of bacterial TRs are $35 \mathrm{kDa}$. The structures of mammalian TRs are similar to those of the other pyridine nucleotide-disulfide oxidoreductases. Most mammalian TRs are selenoproteins containing a rare amino acid, selenocysteine $(\mathrm{U})$, in their C-terminal extensions which have the sequence-GCUG. ${ }^{4}$ High eukaryotic TRs have the various C-terminal sequences shown in Figure 2. They mostly have GCUG at this sequence while Drosophila melanogaster TR has -SCCS and Plasmodium falciparum TR has -GCGGGKCG.

Site-directed modification is useful for the structure-function study of various proteins ${ }^{5-11}$ and is used here to examine the effect on catalytic efficiency of replacement of the C-terminal sequence of human cytosolic TR (-GCUG) with those of Drosophila melanogaster (-SCCS) and Plasmodium falciparum (-GCGGGKCG). The first of these site-directed mutations involved a standard polymerase chain reaction (PCR) using the primers $A$ and $B$, these being respectively (5'-GAAACACCA GGCCGACTCAGAGTAG-3') and (5'-GGGATCCTTAAGAG

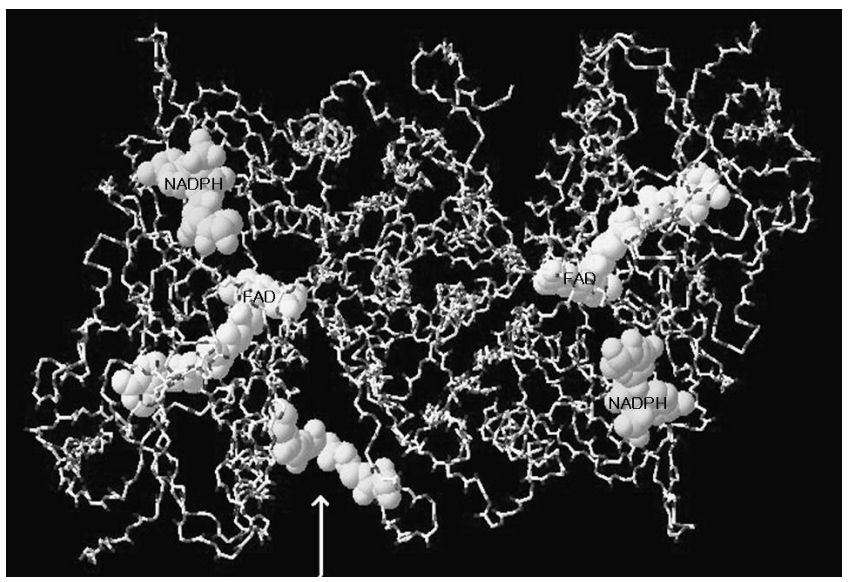

Figure 1. Location of the $\mathrm{C}$-terminal region in a homodimeric rat $\mathrm{TR}$. The FAD, NADPH and C-terminal region (residue-492 to residue499) are shown in space-filled structures and other residues are shown in backbone structures. The C-terminal region is indicated by an arrow.

\begin{tabular}{|c|c|}
\hline 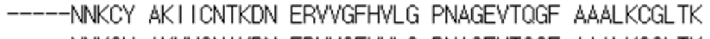 & \\
\hline -NNKCY AKWCNIKDN ERWVGFHVLG PNAGEVTOGF AAALKCGLTK & 462 sp|062768|TRXR1_BOVIN \\
\hline -NNKCY AKI ICNLKDD ERWGFHYLG PNAGEVTOGF AAALKCGLTK & 576 sp|09.JMHG | TRXR1_MOLSE \\
\hline -NNKCY AKVICNLKDN ERWGFHVLG PNAGEVTOGF AAA-KCGLTK & 461 spl089049/TRXR1_RAT \\
\hline -KDHCY LKMICLRNEE EKWGFHILT PNAGGEVTOGF GIALKLAAKK & 629 sp|017745 | TRXR1_CAEEL \\
\hline -VRYCY LKAVAERHGD ORVYGLHYIG PVAGEVIQGF AAALLKSGLTI & 559 sp|P91938|TRXR1_DROME \\
\hline YOLDVSSTCL AKLVVLKNED NRVIGFHYVG PNAGGEVTQGM ALALRLKYKK & 575 sp|P61076 | TRXR2_PLAF7 \\
\hline DSTIGIH PVCAEVFTTL SVTKRSGF & 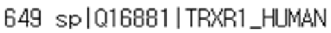 \\
\hline DOLDSTIGIH PVCAEYFTTL SVTKRSGGNI LQ-TGCUG & 499 spl062768 | TRXR1_BOV| \\
\hline OOLDSTIGIH PVCAEIFTTL SVTKRSGGDI LQ-SGCUG & 613 sp|09.JMHG I TRXR1_MOLSE \\
\hline OOLDSTIGIH PVCAEIFTTL SVTKRSGGDI LO-SGCI & 9ITRXR1_RAT \\
\hline ADFDRLIGIH PTVAENFTTL TLEKKEGDEE LQASGCUG & 667 sp|017745 | TRXR1_CAEEL \\
\hline NTL INTVGIH PTTAEEFTRL AI TKRSGLDP TP-ASCCS & 596 sp|P91938 | TRXR1_DROME \\
\hline KDFDNCIGIH PTDAESFMNL FVTISSGLSY AAKGGGCGGGK & 617 splP61076 ITRXR2_PLAF \\
\hline
\end{tabular}

Figure 2. Sequence alignment of C-terminal region of high eukaryotic TRs from various sources (from top to bottom; human, Bos taurus, mouse, Rattus norvegicus, Caenorhabditis elegans, Drosophila melanogaster, and Plasmodium falciparum). Alignment analysis was performed using MAFFT program at ExPASy Proteomics Server (Swiss Institute of Bioinformatics). 
Table 1. Steady state kinetic parameters of mutant and wild-type TRs. The activity was assayed in $10 \mathrm{mM}$ potassium phosphate buffer (pH 7.0) containing $10 \mathrm{mM}$ EDTA, $0.2 \mathrm{mM} \mathrm{NADPH}$, and variable concentrations of DTNB at $25^{\circ} \mathrm{C}$

\begin{tabular}{lccc}
\hline $\begin{array}{c}\text { C-terminal } \\
\text { Sequence }\end{array}$ & $\begin{array}{c}k_{\text {cat }} \\
\left(\mathrm{min}^{-1}\right)\end{array}$ & $\begin{array}{c}K_{m} \text { for DTNB } \\
(\mathrm{mM})\end{array}$ & $\begin{array}{c}k_{\text {cat }} / K_{m} \\
\left(\mathrm{~min}^{-1} / \mathrm{mM}\right)\end{array}$ \\
\hline -GCUG (wild-type) & 1801 & 0.09 & 20011 \\
-SCCS & 192 & 0.47 & 409 \\
-GCGGGKCG & 292 & 0.73 & 400 \\
\hline
\end{tabular}

CAGCAGGAAGCCTG -3': with a BamHI site in bold and mutated bases underlined). The primers are respectively sense and anti-sense oligomers which are used to amplify the whole human TR sequence containing mutations of Gly-497 to Ser (GGC-> TCC) and Gly-499 to Ser (GGC-> TCT). In the second site-directed modification, involving the Plasmodium falciparum sequences, PCRs were carried out using the primers $\mathrm{A}$ (as above), B (5'-TCCTCCTCCGCAGCCAGCCTGGAGGATG CT-3': with inserted bases underlined) and C (5'-GGATCCTTA ACCGACTTTTCCTCCTCCGCAGCCAGCCTG-3': with a Bam HI site in bold and inserted bases underlined). The reaction was performed using the human TR cDNA vector as a template in a programmable PCR machine. ${ }^{5}$ The human TR expression vector which deletes the human TR $\mathrm{Sacl} /$ Bam HI sequence was ligated with the PCR generated human TR SacI/BamHI sequence containing the mutations. The whole inserted sequence was confirmed by DNA sequencing to select the correct human TR mutant expression vector having the intended DNA sequence. The expression of the human TR mutants in $E$. coli was induced by adding IPTG $(1 \mathrm{mM})$. After equilibration with binding buffer, the supernatant solution was applied to the column. E. coli proteins were washed out with binding buffer and then with binding buffer containing $150 \mathrm{mM}$ imidazole. The mutant was eluted with binding buffer containing $500 \mathrm{mM}$ imidazole. Purification was followed by SDS-PAGE (data not shown). The gel showed that the human TR mutant was highly purified.

TR assay was performed by two methods and the activities of the mutants were compared to that of commercially available rat liver TR. A substrate of 5,5'-dithiobis-(2-nitrobenzoate) (DTNB) was used in the first method. The activity of the TR was assayed in $10 \mathrm{mM}$ potassium phosphate buffer ( $\mathrm{pH}$ 7.0) containing 10 mM EDTA, $0.2 \mathrm{mM}$ NADPH, and variable concentrations of DTNB at $25^{\circ} \mathrm{C} .{ }^{12}$ The assay was started by the addition of the enzymes and an increase in absorbance at $412 \mathrm{~nm}$ was observed. The kinetic parameters of the mutants are shown in Table 1 . The $k_{\text {cat }}$ values of the mutants were about $6-9$-fold lower than that of the wild-type rat liver TR, indicating that the catalytic properties of human TR were reduced by the mutations. The $K_{m}$ values of the mutants toward DTNB were about 5- 8fold higher than that of the rat liver TR, indicating that the binding process of DTNB to human TR became less efficient due to the mutations. The $k_{c a} / K_{m}$ values of the mutants toward DTNB were about 50 -fold lower than that of the rat liver TR, indicating that the catalytic efficiencies of the mutants were much decreased by the mutations. The second method employed human recombinant Trx as substrate. The activities of the human TR mutants and rat liver TR were assayed in $100 \mathrm{mM}$ potassium phosphate buffer ( $\mathrm{pH}$ 7.4) containing 2 mM EDTA, $20 \mu \mathrm{M}$ $\mathrm{NADPH}$, and variable concentrations of human $\operatorname{Trx}$ at $25^{\circ} \mathrm{C} .{ }^{12}$ This assay method yielded no trustable activity data of the mutants due to extremely low activity, indicating that the mutations became more detrimental to the enzyme when human Trx was used as a substrate. The $\mathrm{C}$-terminal region of mammalian TRs contains the second redox center and extends the electron transport system from the redox-active disulfide to the enzyme surface. ${ }^{13}$ The C-terminal regions of Drosophila melanogaster (-SCCS) and of Plasmodium falciparum (-GCGGGKCG) also contain the second redox center and are expected to perform the same function. However, they could not replace the $\mathrm{C}$-terminal region of human cytosolic TR. Structural mismatches at the $\mathrm{C}$-terminal region of the mutants could be responsible for this result.

In conclusion, the $\mathrm{C}$-terminal sequence of selenoenzyme human cytosolic TR (-GCUG) was mutated to those of Drosophila melanogaster (-SCCS) and of Plasmodium falciparum (-GCGGGKCG). The mutants were purified and their activities assayed. Both mutants showed much decreased catalytic efficiencies suggesting that the tested alternative $\mathrm{C}$-terminal sequences cannot replace that of human cytosolic TR and improve catalytic efficiency. Also, the conservation of the C-terminal sequence (-GCUG) of the human cytosolic TR is very important to the efficient catalytic function of the enzyme.

Acknowledgments. The authors thank the 21C Frontier Human Gene Bank for providing a human TR cDNA clone. This research was supported in part by the Daegu University Research Grant, 2010.

\section{References}

1. Holmgren, A. Annu. Rev. Biochem. 1985, 54, 237.

2. Williams, C. H., Jr. Enzymes, 3rd ed; Boyer, P., Ed.; Academic Press: 1976; p 89.

3. Williams, C. H., Jr. Eur. J. Biochem. 2000, 267, 6101.

4. Gasdaska, P. Y.; Gasdaska, J. R.; Cochran, S.; Powis, G. FEBS Letters 1995, 373, 5.

5. Kim, H.; Patel, M. S. J. Biol. Chem. 1992, 267, 5128.

6. Yuan, L.; Cho, Y.-J.; Kim, H. Bull. Korean Chem. Soc. 2008, 29, 2327.

7. Yuan, L.; Cho, Y.-J.; Kim, H. Bull. Korean Chem. Soc. 2009, 30, 777.

8. Yoon, M.-Y.; Lee, K.-J.; Kim, J.; Park, H.-C.; Park, S.-H.; Kim, S. G.; Kim, S.-K.; Choi, J.-D.; Bull. Korean Chem. Soc. 2009, 30, 1360.

9. Koh, J.-U.; Cho, H.-Y.; Kong, K.-H. Bull. Korean Chem. Soc. 2007, 28,772 .

10. Park, S. H.; Kim, B. G.; Lee, S. H.; Lim, Y.; Cheong, Y.; Ahn, J.-H. Bull. Korean Chem. Soc. 2007, 28, 2248.

11. Quyen, D. V.; Ha, S. C.; Kim, D.; Lee, S.; Park, S.-J.; Kim, K. K.; Kim, Y.-G. Bull. Korean Chem. Soc. 2007, 28, 2539.

12. Gromer, S.; Arscott, L. D.; Williams, C. H., Jr.; Schirmer, R. H.; Becker, K. J. Biol. Chem. 1998, 273, 20096.

13. Sandalova, T.; Zhong, L.; Lindqvist, Y.; Holmgren, A.; Schneider, G. Proc. Natl. Acad. Sci. USA 2001, 98, 9533. 